\title{
Potential protein post-translational modifications and find potential single amino acid substitutions in hepatitis B large envelope protein
}

\author{
V. Wiwanitkit \\ Department of Laboratory Medicine. Faculty of Medicine. Chulalongkorn University. Bangkok, Thailand
}

\begin{abstract}
RESUMEN
Las modificaciones postranslacionales que sufren las proteínas controlan muchos procesos biológicos. Esto es también válido en el caso de los virus, incluyendo el de la hepatitis B. Sin embargo, no se han realizado estudios en profundidad de la totalidad de la proteína grande de superficie del virus de la hepatitis B. En el presente estudio, se han determinado las modificaciones postranslacionales que son posibles en esta proteína de la cubierta viral empleando para ello una técnica bioinformática estándar. Además, también se determinaron las sustituciones de un solo aminoácido que son posibles en la proteína grande de la superficie del virus de la hepatitis B. De este análisis podemos deducir que existen 3 posibles modificaciones translacionales en esta proteína y una potencial sustitución simple. Estos datos pueden ser útiles tanto para posteriores estudios sobre el virus mutante de la hepatitis $\mathrm{B}$, como para la producción de una nueva vacuna contra este virus.
\end{abstract}

Palabras clave: Modificaciones post-translacionales. Hepatitis B. Proteína de superficie. Sustitución simple.

\begin{abstract}
Post-translational modifications of proteins control many biological processes. This is also important process in virus including hepatitis $\mathrm{B}$. However, there is no in-depth study on the whole hepatitis $\mathrm{B}$ virus large envelope protein. In this work, potential protein post-translational modifications in hepatitis B virus large envelope protein were determined by a standard bioinformatics technique. Furthermore, potential single amino acid substitutions in hepatitis B large envelope protein were also determined. It can be seen that there are possible 3 potential protein were posttranslational modifications with 1 possible single substitution. This data can be useful for further study on hepatitis B virus mutant as well as planning for new vaccine production.
\end{abstract}

Key words: Post-translational modifications. Hepatitis B. Envelope. Single substitution.

Wiwanitkit $V$. Potential protein post-translational modifications and find potential single amino acid substitutions in hepatitis $B$ large envelope protein. Rev Esp Enferm Dig 2008; 100: 153-155.

\section{INTRODUCTION}

Post-translational modifications of proteins control many biological processes, and examining their diversity is critical for understanding mechanisms of cell regulation (1). This is an also important process in virus including hepatitis B (2). Basically, the addition of N-linked glycans to proteins is normally a cotranslational process that occurs during translocation of the nascent protein to the en-

Received: 16-11-07.

Accepted: 27-11-07.

Correspondence: Viroj Wiwanitkit. Department of Laboratory Medicine. Faculty of Medicine. Chulalongkorn University. 10330 Bangkok, Thailand. e-mail: wviroj@yahoo.es doplasmic reticulum (3). Recently, Lambert and Prange reported on an exception to this rule occurring on the hepatitis $\mathrm{B}$ virus large envelope protein that is a subject to co-plus post-translational N-glycosylation (3). Lu et al. also showed evidence that N-linked glycosylation is necessary for hepatitis B virus secretion (4). Sureau et al. also indicated a role of $\mathrm{N}$-glycosylation of hepatitis B virus envelope proteins in morphogenesis and infectivity of hepatitis delta virus (5).

Structurally, the transmembrane topology of a fraction of the large envelope protein $\mathrm{L}$ changes post-translationally, therefore, the $\mathrm{N}$ terminal domain finally appears on both sides of the membrane (6). However, there is no indepth study on the whole hepatitis B virus large envelope protein. In this work, potential protein post-translational 
modifications in hepatitis B virus large envelope protein were determined by a standard bioinformatics technique. Furthermore, potential single amino acid substitutions in hepatitis B large envelope protein were also determined.

\section{MATERIALS AND METHODS}

\section{Obtaining the sequence for hepatitis B large envelope protein}

The database Pubmed (www.pubmed.com) was used for searching for the amino acid sequence for hepatitis B large envelope protein. The derived sequence was used for further predictive study.

\section{Finding for potential protein post-translational modifications and find potential single amino acid substitutions}

The derived amino acid sequence of hepatitis B large envelope protein was further manipulated by bioinformatics tool namely FindMod ${ }^{\mathrm{TM}}$ (Swiss Institute of Bioinformatics, Geneva, Switzerland). Basically, this tool is a tool that can predict potential protein posttranslational modifications and find potential single amino acid substitutions in peptides. It examines peptide mass fingerprinting results of known proteins for the presence of 21 types of post-translational modifications of discrete mass: Acetylation, amidation, biotin, C-mannosylation, deamidation, $\mathrm{N}$-acyl diglyceride cysteine (tripalmitate), flavin adenine dinucleotide (FAD), farnesylation, formylation, geranyl-geranyl, gammacarboxyglutamic acid, O-linked $\beta$-N-acetylglucosamine (O-GlcNAc), hydroxylation, lipoyl, methylation, myristoylation, palmitoylation, phosphorylation, pyridoxal phosphate, pyrrolidone carboxylic acid, sulfatation. This is done by looking at mass differences between experimentally determined peptide masses and theoretical peptide masses calculated from a specified protein sequence (7). This process also provides information about the probability of substitution (7). The points with high probability of substitution will be identified based on the assumption that potential single amino acid substitutions occurring on the cleavage site and substituting the amino acid for an amino acid after which the enzyme used for the digest does not cleave are not displayed (7). This tool is confirmed for reliability and accuracy comparing to mass spectrometry (7). The operative parameters in this study are Tolerance equal to \pm 0.5 Dalton and enzymes are set as trypsin, allowing for up to 3 missed cleavages: a) Cysteine in reduced form, with acrylamide adducts; b) Methionine in oxidized form; and c) Tryptophan in oxidized form.

\section{RESULTS}

\section{Peptide sequences}

According to the searching by the keyword "hepatitis B virus large envelope protein", 14 sequences of large envelope protein of hepatitis B virus could be derived. Of those 14 sequences, only 12 sequences (AAF15903, AAG01538, AAK55756, AAK55755, AAK55754, AAK55753, AAK55752, AAK55751, AAK55750,

\section{AAF15903 \\ mggwsskpqq gmgtnlsvpn pleffpdhql dpafgansnn pdwdfnpnkd hwpeanqvga galgpgftpp hggllgwspq aqgilttvpv apppastnrq sgrqptpisp plrdshpqam qwnsttfhqa lldprvrgly fpaggsssgt vnpvpttasp issifsrtgd papnmentts gflgpllvlq agfslltrih tipqsldsww tsInflggap tcpggnsqsp tsnhsptscp ptcpgyrwmc Irrfiiflfi IIlclifllv Ildyqgmlpv cpllprtstt stgpcktctt paggtsmfps ccctkpsdgn ctcipipssw afarflwewa svrfswlsll vpfvqwfvgl sptvwlsviw imwywgpsly nilspflpll piffclwiyi \\ AAG01538 \\ mggwsskprk gmgtnlsvpn plgffpdhql dpafkanset pdwdlnphkd nwpdankvgv gafgpgftpp hggllgwspq aqgilttvpa appsastnrq sgrqptplsp plrdthpqam kwnsptfhqt lqdprvraly fpaggsssgt vspagntvsa issilsktgd pvpnmenias gllgpllvlq agfflltkil tipgsldsww tsInflggtp vclgqnsqsq isshsptccp picpgyrwmc Irrfiiflci Illclifllv Ildyggmlpv cplipgsstt stgpcktctt pahgtsmfps ccctkptdgn ctcipipssw afakylwewa svrfswlsll vpfvqwfvgl sptvwlsviw mmwywgpsly nilspfmpll piffclwvyi \\ AAF87796 \\ mggwsskprk gmgtnlsvpn plgffpdhql dpafkanset pdwdlnphkd nwpdankvgv gafgpgftpp hggllgwspq aqgilttvpa appsastnrq sgrqptplsp plrdthpqam kwnsptfhqt lqdprvaly fpaggsssgt vspaqntvsa issilsktgd pvpnmenias gllgpllvlq agfflltkil tipqsldsww tsInflggtp vclgqnsqsq isshsptccp picpgyrwmc Irrfiiflci Illclifllv Ildyggmlpv cplipgsstt stgpcktctt pahgtsmfps ccctkptdgn ctcipipssw afakylwewa svrfswlsll vpfvqwfvgl sptvwlsviw mmwywgpsly nilspfmpll piffclwvyi}

Fig. 1. Two studied peptide sequences.

AAK55749, AAK55748, AAF87796), excluding the sequences for hepatitis B virus subtype ADR (AAF05722) and Arctic ground squirrel hepatitis B virus (NP_043865) were selected. From 12 selected sequences, only AAF15903, AAG01538 and AAF87796 had the longest complete codes and used for further analysis (Fig. 1).

Table I. Potential post-translational modifications

\begin{tabular}{lcc}
\hline Potential modification & Peptide & Position \\
\hline DECA & K & 48 \\
OCTA & $R$ & 25 \\
HYPU & VR & $135-136$ \\
\hline
\end{tabular}




\section{Post-translational modifications and potential single amino acid substitutions}

The three selected sequences were analyzed for posttranslational modifications and potential single amino acid substitutions. According to the analysis, the same results could be observed. For each sequence, 3 post-translational modifications could be identified (Table I). Concerning single amino acid substitutions prediction, only point with the high probability for substitution could be seen. $\mathrm{V} \rightarrow \mathrm{W}$ at peptide V (position 135) could be determined.

\section{DISCUSSION}

The hepatitis B virus envelope and the subviral lipoprotein particles contain three viral surface proteins (L, M, and S) which are expressed from one open reading frame by the usage of three start codons and a common stop codon. It adopts two different transmembrane topologies due to a posttranslational switch of the folding in approximately half of the proteins (8). Post-translational modifications of hepatitis $\mathrm{B}$ virus envelope protein are reported and becomes a new focus of hepatitis B research. It is hypothesized that failure to process $\mathrm{N}$-glycan causes HBV glycoproteins to aggregate and that impaired protein-protein interactions and trafficking are the result of misfolding (9). This process is related to further mutated or variant virus. The presence of pre-S1, pre-S2, and S epitopes at the outer surface confirmed that mutant viruses were normally assembled (10). By contrast, myristylation-defective mutants completely lost their infectivity for human hepatocytes in primary cultures as shown by the absence of HBs antigen production and viral intermediate replicative forms in hepatocytes (10). The modifications and substitutions can be the factor contributing to changes in viral genotype, immunological response, serotypes, clinical expression, prognosis, response to antiviral therapy and development of hepatocellular carcinoma (11-14).

In the previous day, it is hard to study or predicted potential protein post-translational modifications. However, it is presently possible with use of novel bioinformatics tool. In this work, the author predicted potential protein post-translational modifications and find poten- tial single amino acid substitutions in hepatitis B large envelope protein. A new tool was used. It can be seen that there are possible 3 potential protein post-translational modifications with 1 possible single substitution. For the predicted substitution, it has never been reported in the literature. The effects due to possible changes in the identified points require further research to document. This data can be useful for further study on hepatitis B virus mutant as well as planning for new vaccine production. Selection of epitope candidate to cover these regions is necessary.

\section{REFERENCES}

1. Witze ES, Old WM, Resing KA, Ahn NG. Mapping protein posttranslational modifications with mass spectrometry. Nat Methods 2007; 4 (10): 798-806.

2. Bruss V. Processing of hepatitis B virus surface proteins. Methods Mol Med 2004; 95: 189-98.

3. Lambert C, Prange R. Posttranslational N-glycosylation of the hepatitis B virus large envelope protein. Virol J 2007; 4: 45.

4. Lu X, Mehta A, Dwek R, Butters T, Block T. Evidence that N-linked glycosylation is necessary for hepatitis B virus secretion. Virol 1995; 213 (2): 660-5.

5. Sureau C, Fournier-Wirth C, Maurel P. Role of N glycosylation of hepatitis $\mathrm{B}$ virus envelope proteins in morphogenesis and infectivity of hepatitis delta virus. J Virol 2003; 77 (9): 5519-23.

6. Bruss V. Hepatitis B virus morphogenesis. World J Gastroenterol 2007; 13 (1): 65-73.

7. Wilkins MR, Gasteiger E, Gooley AA, Herbert BR, Molloy MP, Binz $\mathrm{PA}$, et al. High-throughput mass spectrometric discovery of protein post-translational modifications. J Mol Biol 1999; 289 (3): 645-57.

8. Bruss V, Gerhardt E, Vieluf K, Wunderlich G. Functions of the large hepatitis B virus surface protein in viral particle morphogenesis. Intervirol 1996; 39 (1-2): 23-31.

9. Lu X, Mehta A, Dadmarz M, Dwek R, Blumberg BS, Block TM. Aberrant trafficking of hepatitis B virus glycoproteins in cells in which N-glycan processing is inhibited. Proc Natl Acad Sci USA 1997; 94 (6): 2380-5.

10. Gripon P, Le Seyec J, Rumin S, Guguen-Guillouzo C. Myristylation of the hepatitis B virus large surface protein is essential for viral infectivity. Virol 1995; 213 (2): 292-9.

11. Sureau $C$. The role of the HBV envelope proteins in the HDV replication cycle. Curr Top Microbiol Immunol 2006; 307: 113-31.

12. Cougot D, Neuveut C, Buendía MA. HBV induced carcinogenesis. J Clin Virol 2005; 34 (Supl. 1): S75-8.

13. Chisari FV. Hepatitis B virus transgenic mice: Insights into the virus and the disease. Hepatol 1995; 22 (4 Pt 1): 1316-25.

14. Chisari FV. Hepatitis B virus transgenic mice: Models of viral immunobiology and pathogenesis. Curr Top Microbiol Immunol 1996; 206: $149-73$. 